\title{
Hypoxia imaging in upper gastrointestinal tumors and application to radiation therapy
}

\author{
Randa Tao ${ }^{1}$, Bryan Ager ${ }^{1}$, Shane Lloyd ${ }^{1}$, Anna Torgeson ${ }^{1}$, Michelle Denney ${ }^{1}$, David Gaffney ${ }^{1}$, Jordan \\ Kharofa $^{2}$, Steven H. Lin ${ }^{3}$, Albert C. Koong ${ }^{3}$, Yoshimi Anzai ${ }^{4}$ John M. Hoffman ${ }^{5}$ \\ ${ }^{1}$ Department of Radiation Oncology, Huntsman Cancer Institute, University of Utah, Salt Lake City, UT, USA; ${ }^{2}$ Department of Radiation Oncology, \\ University of Cincinnati, Cincinnati, OH, USA; ${ }^{3}$ Department of Radiation Oncology, The University of Texas MD Anderson Cancer Center, \\ Houston, TX, USA; ${ }^{4}$ Department of Radiology, ${ }^{5}$ Center for Quantitative Cancer Imaging, Huntsman Cancer Institute, University of Utah, Salt Lake \\ City, UT, USA \\ Contributions: (I) Conception and design: R Tao, S Lloyd, D Gaffney, JM Hoffman; (II) Administrative support: M Denney; (III) Provision of study \\ materials or patients: R Tao, B Ager, A Torgeson, M Denney, J Kharofa, AC Koong; (IV) Collection and assembly of data: R Tao, B Ager; (V) Data \\ analysis and interpretation: R Tao, B Ager, S Lloyd, A Torgeson, D Gaffney, J Kharofa, SH Lin, AC Koong, Y Anzai, JM Hoffman; (VI) Manuscript \\ writing: All authors; (VII) Final approval of manuscript: All authors. \\ Correspondence to: Randa Tao, MD. Department of Radiation Oncology, Huntsman Cancer Hospital, University of Utah, 1950 Circle of Hope, Room \\ 1570, Salt Lake City, UT 84112, USA. Email: randa.tao@hci.utah.edu.
}

\begin{abstract}
Survival for upper gastrointestinal tumors remains poor, likely in part due to treatment resistance associated with intratumoral hypoxia. In this review, we highlight advances in nuclear medicine imaging that allow for characterization of in vivo tumor hypoxia in esophageal, pancreatic, and liver cancers. Strategies for adaptive radiotherapy in upper gastrointestinal tumors are proposed that would apply information gained through hypoxia imaging to the creation of personalized radiotherapy treatment plans able to overcome hypoxia-induced treatment resistance, minimize treatment-related toxicities, and improve patient outcomes.
\end{abstract}

Keywords: Hypoxia; fluorine-18 fluoromisonidazole positron-emission tomography (FMISO PET); radiotherapy; esophagus cancer; pancreas cancer; liver cancer

Submitted Apr 27, 2018. Accepted for publication Sep 20, 2018.

doi: 10.21037/jgo.2018.09.15

View this article at: http://dx.doi.org/10.21037/jgo.2018.09.15

\section{Introduction}

Malignant tumors have complex intratumor heterogeneity with distinct patterns of growth, gene expression, blood flow, oxygenation, and metabolism throughout different portions of the tumor. Some of these characteristics of the tumor microenvironment may be exploited therapeutically for cancer therapy (1-3). Intratumor heterogeneity increases as cancer cells proliferate and a tumor grows in size (4). This leads to the formation of subpopulations of cells that exhibit varying levels of proliferative ability and spatially distinct tumor microenvironments that may ultimately drive treatment resistance (5). Hence, an attractive concept in cancer research is to use advanced imaging techniques to assess the heterogeneity of tumor physiology, metabolism, proliferation, hypoxia and other biologic properties, and correlate the imaging findings with response and outcomes.

Hypoxia is an example of a measurable property of tumor biology associated with increased tumor aggressiveness, potential for metastatic spread, and resistance to therapy that translates into worse survival outcomes for patients (6-8). Because the extent of tumor hypoxia does not necessarily correlate with tumor size or grade, investigators have looked toward molecular and imaging biomarkers of hypoxia (9). Several nitroimidazole derivatives have been identified as hypoxia radiotracers with positron-emission tomography (PET) imaging. The basic mechanism of action involves stabilization with nitroreductase enzymes resulting in cellular accumulation of the nitroimidazole compound under hypoxic conditions, allowing visualization and 


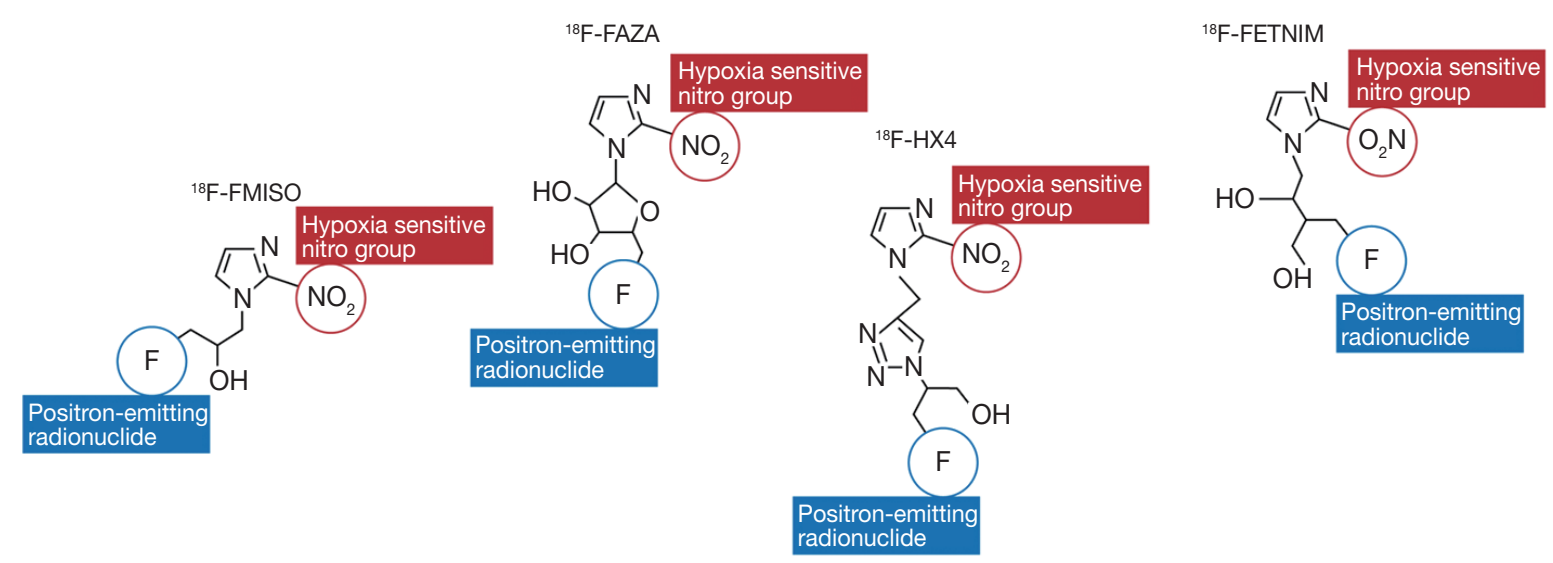

Figure 1 Nitroimidazole family of hypoxia positron-emission tomography (PET) tracers. Chemical structure of fluorine-18 fluoromisonidazole (FMISO), fluorine-18 fluoroazomycin arabinoside (FAZA), fluorine-18-3-fluoro-2-\{4-[(2-nitro-1H-imidazol-1-yl) methyl]-1H-1,2,3-triazol-1-yl\}propan-1-ol (HX4), and fluorine-18 fluoroerythronitroimidzaole (FETNIM). The nitro group (NO ${ }_{2}$ ) on each compound is targeted by nitroreductase enzymes in hypoxic conditions resulting in cellular accumulation. The nitroimidazole compound is labeled with fluorine-18 (F), the positron-emitting radionuclide, for visualization on PET imaging.

quantification of the areas and extent of tumor hypoxia (10). The labeling of the nitroimidazole compound with fluorine-18 then identifies hypoxic regions via PET imaging. One such nitroimidazole derivative is fluorine- 18 fluoromisonidazole (FMISO), which was first developed in 1986, and has been shown to specifically image hypoxic areas in many tumor types $(11,12)$. As it is a relatively older radiotracer, it has been more established as a hypoxia marker in the clinical setting compared to other nitroimidazole derivatives such as fluorine-18 fluoroazomycin arabinoside (FAZA), and fluorine-18 fluoroerythronitroimidzaole (FETNIM). The chemical structure of select nitroimidazole compounds are shown in Figure 1. Increased uptake of FMISO has been correlated with worse prognosis in lung cancer and head and neck cancer patients (13). Furthermore, it has been demonstrated as a predictor of treatment response to radiation therapy with the most data in head and neck cancers $(14,15)$.

Upper gastrointestinal tumors are an area with poor outcomes with potential benefit from hypoxia guided radiation therapy. Although neoadjuvant chemoradiation therapy has been shown to significantly improve overall survival in the landmark CROSS trial for esophageal cancer, the majority of patients did not experience a pathologic complete response (16). This was especially true for adenocarcinomas and overall prognosis remains poor. The pathologic response rates of pancreas cancers are even lower to chemoradiation therapy $(17,18)$ and a clinically significant portion of patients die from complications of their local disease (19). Moreover, the proximity to relatively radiosensitive organs such as bowel or stomach limits the radiation dose that may be used to treat these tumors. Tumor microenvironmental factors, such as hypoxia, that reduce the effectiveness of radiation further compromises the delivery of curative radiation doses to this area of the body. Thus, there is a clear need to improve local treatment options in upper gastrointestinal cancers. As hypoxia is a known mechanism of radiation resistance, it may partly explain the poor response to radiation therapy observed in gastrointestinal adenocarcinomas. Experimental studies have consistently shown that cells irradiated under hypoxic conditions are $2-3$ times more resistant to radiation therapy $(20,21)$. This is thought to be secondary to a lack of oxygen which, when present, allows for the generation of free radicals that are thought to be responsible for the majority of indirect DNA damage and cell kill from ionizing radiation. An attractive method to improve response to radiation therapy includes identifying the radioresistant hypoxic regions and potentially targeting them with a higher radiation dose (22).

In this review, we summarize the state of known and experimental imaging markers of hypoxia and how they have been studied in esophagus, pancreas, and liver cancers. The purpose of this manuscript is to review studies done with hypoxia imaging in patients with these tumors. Most studies done for each tumor site will be summarized or referenced but this manuscript is not meant to be a systematic review of the available literature. It is meant to highlight the state 


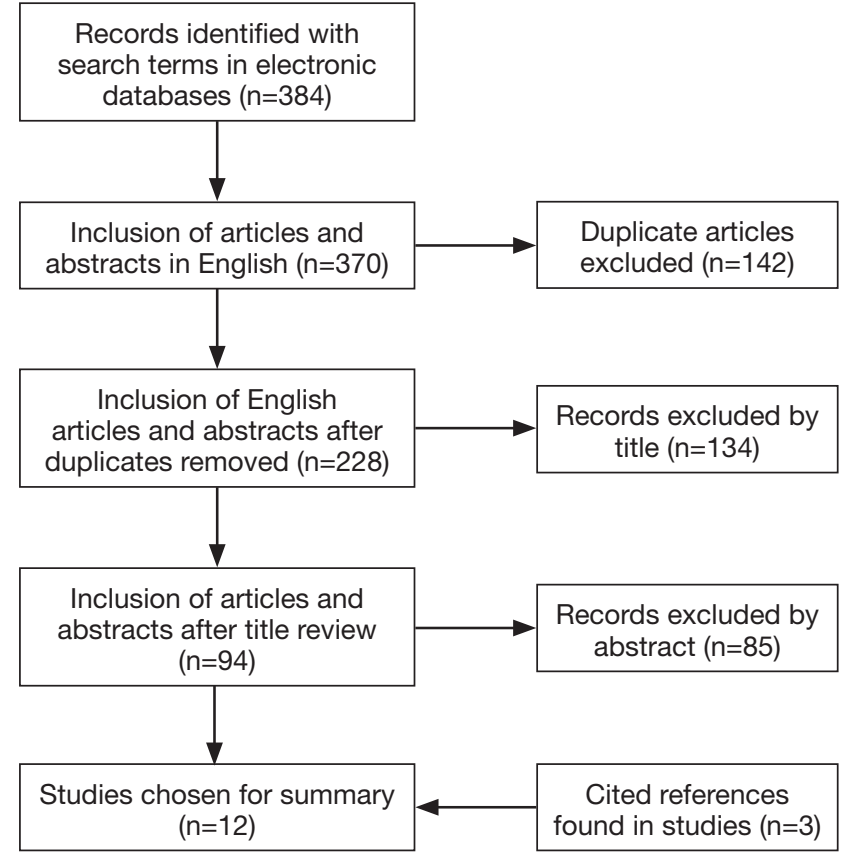

Figure 2 Flow diagram illustrating the selection of articles summarized.

of the known imaging techniques in order to propose how they can be applied to the field of radiation oncology. We then discuss the potential strategies that can be employed in the clinic with hypoxia imaging to improve the delivery of radiation therapy.

\section{Methods}

A literature search was performed using PubMed (National Center for Biotechnology Information, NCBI), Google Scholar, and MEDLINE (U.S. National Library of Medicine). Search terms used included "hypoxia," "imaging," "esophagus cancer," "pancreas cancer," "liver cancer," "hepatocellular carcinoma," "intrahepatic cholangiocarcinoma," "radiation," "chemoradiation," and "radiotherapy." These search terms were also used to identify active clinical trials on the ClinicalTrials.gov website. English-language original articles, abstracts, and reviews were included. A flow diagram illustrating the identified articles with the search terms is shown in Figure 2. Articles were excluded if the imaging techniques used in the study did not include known hypoxia markers, if they were pure pre-clinical models without a corresponding study done in patients, or if they did not include the appropriate tumor sites. An exception was made for an article that studied an animal model of liver tumors. This article was referenced given the limited amount of literature available in patients. Review articles were used in this search to further identify original articles that were included in this summary. The highlighted studies described are summarized in Table 1.

\section{Tumor hypoxia imaging}

\section{Esophagus cancer}

Tumor hypoxia has been demonstrated in esophageal cancer patients, and interestingly, increased hypoxia has been demonstrated in patients with adenocarcinomas over squamous cell carcinomas. In one study of 20 patients, mean FMISO accumulation was significantly higher in adenocarcinomas relative to squamous cell carcinomas, whereas glucose metabolism did not differ by histology (23). These findings were confirmed by a follow-up study of 38 patients that demonstrated significant tumor hypoxia in $87 \%$ of esophageal cancers imaged with FMISO PET, again showing higher relative uptake seen with adenocarcinoma histology (24). It was hypothesized that this could partly explain the lower pathologic complete response rates observed in adenocarcinomas compared to squamous cell carcinomas. In a cohort of untreated squamous cell esophageal carcinoma patients, investigators from China demonstrated the feasibility of FETNIM PET in detecting tumor hypoxia and were able to correlate FETNIM maximum standardized uptake values (SUVmax) with a poorer clinical response to treatment with concurrent chemoradiation therapy (25). A newer nitroimidazole radiotracer 18F-3-fluoro-2-\{4-[(2-nitro-1H-imidazol-1-yl) methyl]-1H-1,2,3-triazol-1-yl\}propan-1-ol (HX4) has also shown promise in reproducibly visualizing esophageal tumor hypoxia while affording potential pharmacokinetic advantages over other nitroimidazole derivatives as well as less spatiotemporal variability in tracer uptake (26). In this study, HX4 was shown to have repeatable uptake with a high tumor-to-background ratio in a study of 19 esophagus cancer patients with both adenocarcinoma and squamous histologies. While other PET radiotracers such as FAZA, $\mathrm{Cu}(\mathrm{II})$-diacetyl-bis(N4-methylthiosemicarbazone) $(\mathrm{Cu}-$ ATSM), and fluorine-18 fluoroetanidazole (FETA) also have potential to image tumor hypoxia, none have yet been studied in esophageal cancer (30). Techniques to image hypoxia with magnetic resonance imaging (MRI) have also 


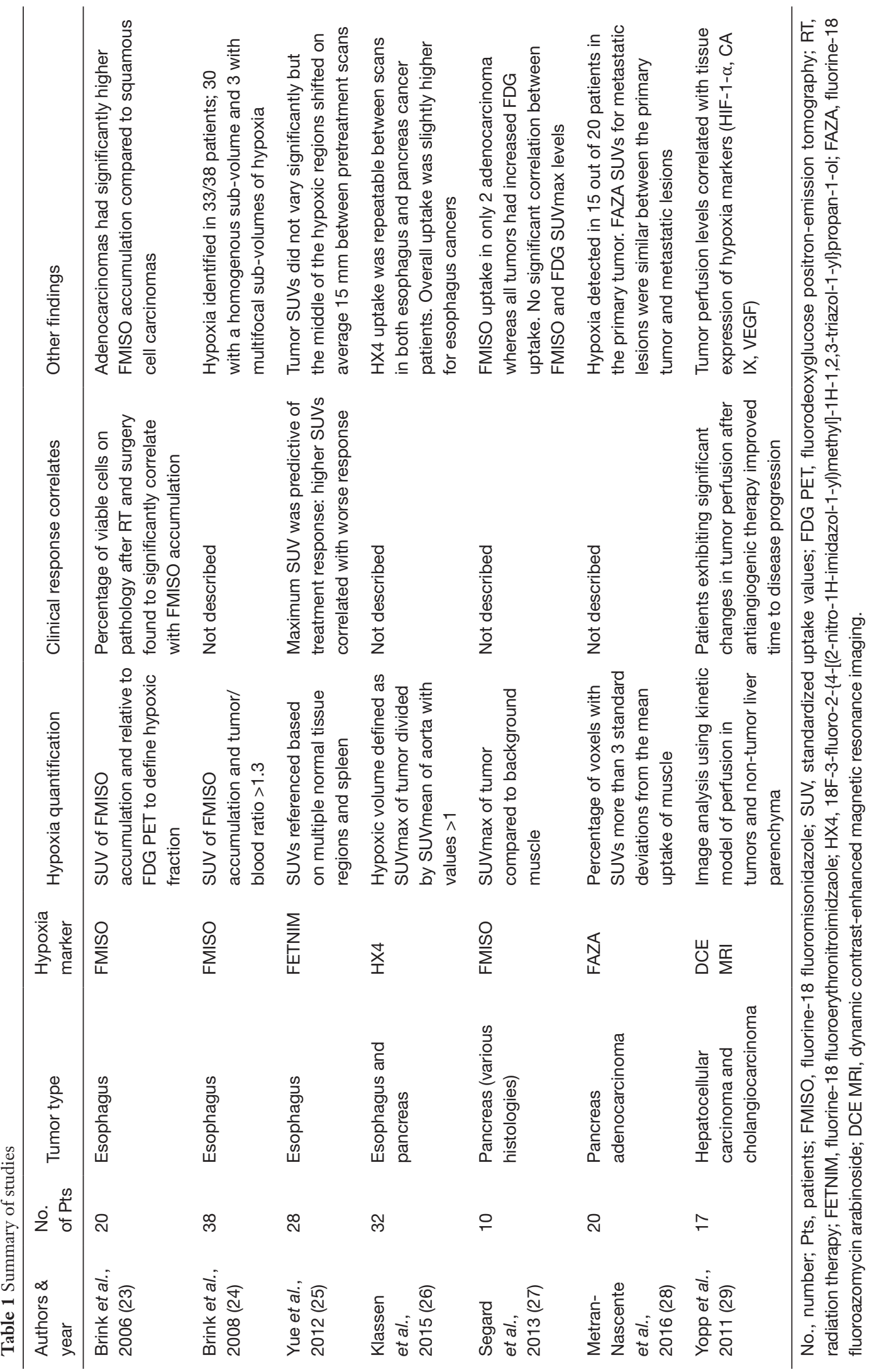


not yet been evaluated in esophageal cancer patients.

\section{Pancreas cancer}

The hypoxic pancreatic tumor microenvironment, characterized by reduced vascularity and an abundance of stromal tissue, likely contributes to an aggressive clinical course and reduced responsiveness to treatment (31-34). A specific example of the pancreas tumor microenvironment contributing to disordered vascularity includes findings that the tumor stroma contains high levels of hyaluronic acid, which raises the interstitial pressure and decreases perfusion in the tumor (35). In spite of these hypoxic conditions, FMISO demonstrated minimal activity in detecting hypoxia in 10 patients with a variety of pancreatic tumor histologies, including 7 with pancreatic ductal adenocarcinomas, 1 neuroendocrine carcinoma, 1 poorly differentiated/sarcomatoid carcinoma, and 1 mucinous neoplasm (27). In a study evaluating 20 patients with PET FAZA imaging, hypoxia was only detected in $75 \%$ percent of patients, and the hypoxic fraction ranged from less than $5 \%$ to greater than $50 \%$, suggesting that the tumor microenvironment of pancreatic adenocarcinomas may not be as uniformly hypoxic as once thought (28). In contrast to these experiences, PET imaging with HX4 was found to feasibly and reliably visualize pancreatic tumor hypoxia in a cohort of 13 patients (26). This discrepancy was attributed to faster background clearance with $\mathrm{HX} 4$ relative to other nitroimidazole derivatives as well as to the longer time interval between radiotracer injection and scanning used in the study. Thus, detection and characterization of hypoxia may be dependent upon the imaging modality used and the presence of severe hypoxia in pancreatic cancer likely contributes to the aggressive biologic behavior of these tumors. In an effort to better understand the diagnostic value and predictive potential for hypoxia imaging in pancreatic cancer, a single-arm single institution prospective clinical trial is attempting to correlate PET FAZA uptake with extent of disease, serum biomarkers, treatment response, and survival endpoints in pancreatic cancer (clinicaltrials.gov: NCT01542177). MRI techniques such as dynamic contrast enhancement $(36,37)$ or diffusion weighted imaging (38) are also under study to evaluate tumor hypoxia in pancreatic cancer.

\section{Liver cancer}

Due to physiologic hepatic metabolism of nitroimidazole

(C) Journal of Gastrointestinal Oncology. All rights reserved. derivatives, few in vivo studies have evaluated hypoxia imaging biomarkers in liver tumors. However, within a preclinical orthotopic rat model, FMISO and iodine-124 iodoazomycin galactopyranoside (IAZG) were both found to detect tumor hypoxia in implanted liver tumors and peritoneal metastases (39). Higher contrast ratios and superior diagnostic image quality were observed with FMISO compared to IAZG. In a phase II study of 17 patients with hepatocellular carcinoma or intrahepatic cholangiocarcinoma, Yopp and colleagues utilized dynamic contrast-enhanced magnetic resonance imaging (DCE$\mathrm{MRI})$ to measure the degree of tumor re-oxygenation after treatment with floxuridine (FUDR) and bevacizumab (29). The rationale for using the antiangiogenic agent bevacizumab was that it could potentially reverse tumor hypoxia and improve the delivery of cytotoxic chemotherapy, which in this case was FUDR. The investigators also stained tumor tissue for baseline expression of 3 hypoxia markers: anti-hypoxia inducible factor-1 $\alpha$ (HIF-1 $\alpha$ ), anti-carbonic anhydrase IX (CA IX), and vascular endothelial growth factor (VEGF). DCE-MRI parameters correlating with tumor perfusion were found to correlate with baseline tissue markers of hypoxia as well as time to progression in these patients with primary liver cancers. Interestingly, only patients with tumors expressing these hypoxia markers exhibited significant changes in the DCE-MRI perfusion parameters after bevacizumab therapy. Additionally, improvement in clinical outcomes with a longer time to progression was observed only in patients who exhibited significant changes in DCE-MRI parameters after administration of bevacizumab. The authors hypothesize that these changes in DCE-MRI parameters could reflect normalization of tumor vasculature leading to decreased hypoxia and conclude that DCE-MRI may be a useful biomarker of therapeutic response, especially after treatment with antiangiogenic therapy. However, a concern with DCEMRI imaging is that it provides only an indirect measure of hypoxia that uses blood flow as a surrogate $(40,41)$. As multiple factors influence the degree of hypoxia in tumors, blood flow may not always correlate with tumor hypoxia. Although experience is limited, these studies suggest that hypoxia imaging is feasible in primary liver cancers and could also be predictive of treatment response and clinical outcomes. Additional studies are warranted to develop novel imaging biomarkers that can better characterize in vivo tissue hypoxia within metastatic and primary tumors of the liver. 


\section{Strategies to integrate hypoxia imaging with radiation therapy}

One strategy is to apply the information gained from hypoxia imaging and use it to individualize total radiation dose or to adapt the radiation dose during treatment based on initial response. For example, patients with tumors exhibiting hypoxia could be selected for dose escalation or de-escalation if their tumors exhibit an early response to radiation therapy. In a seminal pilot study from Lee $e t$ al., 33 patients with HPV positive oropharyngeal cancer were evaluated for pretreatment hypoxia with FMISO (defined as $>1.2$ tumor to muscle standardized uptake value) (42). A repeat scan was performed one week after initiating definitive chemoradiation. Interestingly, 100\% of patients demonstrated pretreatment hypoxia of either the primary site or involved regional lymph nodes. However, hypoxia resolved within one week of treatment in $48 \%$ of cases. Patients with resolution of hypoxia after one week of treatment received a 10 Gray (Gy) radiotherapy dose reduction to metastatic lymph node(s) from 70 to $60 \mathrm{~Gy}$. For the entire cohort, including patients who underwent adaptive dose reduction, $100 \%$-year local control was achieved with $100 \% 2$-year overall survival. One patient with persistent hypoxia on FMISO PET developed distant metastatic disease, corresponding to a 2-year distant metastases-free survival rate of $97 \%$. Of course, it is not known whether these excellent outcomes would have been observed without this adaptive radiation dosing technique; $100 \% 2$-year local control and overall survival may have been observed if all patients were treated to $60 \mathrm{~Gy}$ as HPV positive oropharyngeal cancers are known to be sensitive to radiation therapy. In addition, hypoxia imaging only gives a snapshot of a dynamic process and is thus subject to sampling error. Variations in hypoxic imaging of normal tissues may also suggest differential normal tissue toxicities between patients. Nonetheless, these promising results suggest that adaptive radiotherapy based on hypoxia imaging is potentially safe and feasible, offering a new avenue for further personalizing oncologic therapy and minimizing unnecessary treatment-related toxicities.

In light of this intriguing potential, adaptive radiotherapy via advanced imaging has gained acceptance in the treatment of other cancers. In a phase II clinical trial by Kong et al., midtreatment standard fluorine-18 fludeoxyglucose (FDG) PET was acquired in 42 patients with inoperable stage II-III non-small cell lung cancer (NSCLC). Based on the degree of intra-treatment PET metabolic activity, radiotherapy was adaptively dose-escalated up to a maximum of 86 Gy in 30 fractions, with a threshold of grade $>2$ radiation-induced lung injury (43). Concurrent weekly carboplatin and paclitaxel were given, as well as three cycles of consolidation chemotherapy. At a median follow-up of 47 months, 2-year infield and overall locoregional control rates were $82 \%$ and $62 \%$, respectively. Median overall survival was 25 months with 2- and 5-year overall survival rates of $52 \%$ and $30 \%$, respectively. These local control and survival rates compare favorably to historical controls with standard chemoradiation, suggesting yet another exciting possibility for improving patient outcomes with individualized, response-based adaptive radiotherapy. It is interesting that the landmark RTOG 0617 randomized trial failed to show improved tumor control with higher doses of radiation therapy (44), which may be partly explained by applying uniform dose escalation to unselected patients.

FDG PET may be a feasible imaging tool to guide dose escalation in radiation therapy, but studies have shown that it is actually not a reliable imaging correlate for hypoxia $(45,46)$. Thus, there is a need to investigate further the role of hypoxia imaging to determine which patients are best-selected for radiotherapy dose-escalation and whether hypoxic portions of a tumor can be selectively targeted in order to overcome radioresistance. One simple strategy is to select patients with tumors exhibiting a high degree of hypoxia and uniformly increase radiation dose to the entire extent of the gross tumor volume (GTV). However, this strategy often results in unacceptably high dose to the surrounding normal tissues. With advances in radiation technology and planning, including intensity-modulated radiation therapy (IMRT) and stereotactic body radiation therapy (SBRT), heterogeneous dosing to tumors with dose-painting is now possible. Therefore, an alternative strategy would be to use hypoxia imaging to selectively boost only the hypoxic regions of the tumor, which may make it possible to avoid increasing dose to normal tissues $(22,47)$. Several modelling studies based on established principles in radiobiology suggest that an increase in total radiation dose to the hypoxic region of a tumor can significantly increase tumor control probability. Although hypoxic cells are considered 2-3 times more radioresistant, one study used a Monte Carlo model to show that only a moderate increase in dose of $120-150 \%$ to areas of chronic hypoxia led to a significant increase in tumor control probability (48). Another study in head and neck cancer patients used FMISO PET to determine the target of an added simultaneous integrated boost (SIB) of 10 Gy 
to each patient's IMRT plan and demonstrated a mean improvement in tumor control probability of $17 \%$ without a significant associated increase in normal tissue complication probability (49).

There is a clear rationale to apply these strategies in the radiation treatment of patients with upper gastrointestinal tumors. Similar to the results in the lung cancer literature, dose escalation in unselected patients with esophagus cancer has not been shown to have a benefit on local control or survival (50). For pancreas and liver tumors, conventional doses of radiation therapy have significant limitations in achieving disease control with clinical evidence suggesting that higher doses may lead to improved outcomes (51-55). Upper gastrointestinal tumors are especially challenging due to the requirement for higher radiation dose for disease control, yet close anatomic proximity to exquisitely radiation-sensitive organs such as the small bowel and stomach. In clinical practice, a boost of higher radiation dose may be given to the portion of pancreatic tumors away from the dose-limiting duodenum, or to an arbitrary central region of a liver tumor assumed to harbor hypoxic radioresistant cells $(51,52)$. However, no study has been done to identify the true areas of hypoxia that could more accurately be used to define and individualize the radiation dosing specific to each patient's tumor. Thus, these noninvasive hypoxia tracers could be used to rationally define radiation treatment volumes by more accurately individualizing radiation dosing specific to each unique tumor microenvironment.

Although FMISO, FETNIM, and HX4 have not been directly compared in esophageal cancer, a reasonable next step in designing a clinical study would be to pick one tracer to use in a pre-treatment and mid-treatment scan and selectively design dose-escalated radiotherapy plans for patients with hypoxic tumors. In this concept, the hypoxic imaging assessment would be an integral biomarker in defining different therapy arms. In operable patients, the pathologic response could be correlated with the degree of pre- and mid-treatment hypoxia. The challenge is in determining how much the selective or total GTV boosting versus baseline tumor biology contributed to the pathologic and/or clinical response.

For pancreatic cancers, some small initial studies showed that FMISO and FAZA were not reliable markers of hypoxia in pancreas tumors, but HX4 appears to show the most promise. Thus, HX4 could be used to determine the feasibility of a selected hypoxia-targeted pancreatic boost. Whether this decreases in the maximum and volumetric doses to the dose-limiting structures in pancreas cancer to allow for safer and individualized dose escalation can then be investigated. Additional clinical data is needed to demonstrate the feasibility of this approach.

The next step for liver tumors is to apply the pre-clinical data to the clinical setting in patients with hepatocellular carcinoma, intrahepatic cholangiocarcinoma, and potentially patients with metastatic disease to the liver. FMISO was shown to have superior imaging quality than IAZG in the preclinical data and could be investigated as one of the first radiotracers clinically assessing hypoxia in patients with liver tumors and in determining if any correlates to standard-of-care imaging exist. For example, a pilot study could assess whether there is correlation between FMISO uptake and the degree of enhancement on MRI for hepatocellular carcinoma or FDG PET for intrahepatic cholangiocarcinoma and metastatic tumors. Mid- and posttreatment FMISO scans could be done to assess the degree of change in tumor hypoxia during radiation therapy and to assess whether these imaging findings are predictive of treatment response and clinical outcomes. This information could then be used in radiation treatment planning to design an individualized boost volume based on real-time hypoxic volumes, rather than arbitrarily targeting the central tumor volume that may not correlate with true areas of hypoxia. This strategy could spare unnecessary radiation dose to the normal liver parenchyma, while still providing treatment intensification to potentially radioresistant regions of tumor.

For gastrointestinal cancer patients receiving neoadjuvant chemotherapy, it would be of interest to investigate which systemic agents can potentially reverse tumor hypoxia with the aid of hypoxic imaging. Many antivascular targeted agents are now available, yet their effect on hypoxia in gastrointestinal malignancies is not well defined. Research in these areas could permit a further personalized approach in the selection of chemotherapy.

There are recognized challenges in translating these imaging techniques to the clinic. In order to implement hypoxia imaging information into IMRT treatment planning, the spatial resolution of imaging has to be sufficient to differentiate subregion of hypoxia from the rest of normoxic tumor. Accurately defining the spatial and temporal variation in the areas of tumor hypoxia throughout the course of treatment is particularly challenging. To fully characterize this variation, a hypoxia radiotracer scan would have to be done before or after each fraction of radiation therapy. These multiple sets of imaging data would then need to be compared to quantify the extent of variability 
both within and between patients. This may be practical for a short course of radiation therapy, such as a 3 - or 5 -fraction SBRT treatment schedule, but becomes impractical with longer courses of therapy. Thus, an unanswered question is how to best integrate potential changes in the hypoxic areas of tumors into the radiation plan throughout the course of treatment, particularly if selective boosting is to be used. It would be impractical to perform a scan before every treatment. Perhaps weekly or mid-treatment scans would provide enough information. Another mechanism of integrating hypoxia imaging with radiation therapy includes the use of MRI-based linear accelerators. With MRI-based linear accelerators, it may be more practical for patients to be imaged daily and MRI-based biomarkers for hypoxia could be incorporated into adaptive radiation protocols. This would potentially allow for more frequent assessment of hypoxia in a practical manner. The optimal frequency of hypoxia imaging scans during radiotherapy requires further investigation.

\section{Summary and future directions}

Studies have consistently shown that tumors exhibiting hypoxia are associated with poor prognosis including a worse response to radiation therapy. Imaging with PET radiotracers, particularly the nitroimidazoles, is a reproducible method of visualizing areas of tumor hypoxia that could be used to select patients for different methods of radiation dose escalation or adaptation. Future studies are needed to establish whether disease outcomes can be improved with increasing radiation dose to hypoxic tumors. Given that many patients with upper gastrointestinal tumors may be unable to proceed with surgery due to the advanced nature of their local disease or medical comorbidities, the role of radiotherapy becomes even more important for local control and possibly survival. Unfortunately, responses to radiotherapy or even chemoradiation therapy have been poor. Dose escalation to entire treatment volumes have been either ineffective or are quite difficult due to risk of damage to surrounding organs and tissue. One possibility for making radiotherapy more effective is by selectively targeting hypoxic regions of tumor with higher doses of radiotherapy, thus affecting more direct tumor cell kill. Adaptive treatment algorithms utilizing hypoxia as a biomarker has significant promise to personalize chemotherapy and radiation therapy in a tumorspecific fashion and improve outcomes in gastrointestinal malignancies.

\section{Acknowledgements}

None.

\section{Footnote}

Conflicts of Interest: The authors have no conflicts of interest to declare.

\section{References}

1. Rankin EB, Giaccia AJ. Hypoxic control of metastasis. Science 2016;352:175-80.

2. LaGory EL, Giaccia AJ. The ever-expanding role of HIF in tumour and stromal biology. Nat Cell Biol 2016;18:356-65.

3. Jiang D, Niwa M, Koong AC. Targeting the IRE1alphaXBP1 branch of the unfolded protein response in human diseases. Semin Cancer Biol 2015;33:48-56.

4. Eskey CJ, Koretsky AP, Domach MM, et al. 2H-nuclear magnetic resonance imaging of tumor blood flow: spatial and temporal heterogeneity in a tissue-isolated mammary adenocarcinoma. Cancer Res 1992;52:6010-9.

5. Junttila MR, de Sauvage FJ. Influence of tumour microenvironment heterogeneity on therapeutic response. Nature 2013;501:346-54.

6. Harris AL. Hypoxia--a key regulatory factor in tumour growth. Nat Rev Cancer 2002;2:38-47.

7. Dewhirst MW, Cao Y, Moeller B. Cycling hypoxia and free radicals regulate angiogenesis and radiotherapy response. Nat Rev Cancer 2008;8:425-37.

8. Nordsmark M, Bentzen SM, Rudat V, et al. Prognostic value of tumor oxygenation in 397 head and neck tumors after primary radiation therapy. An international multicenter study. Radiother Oncol 2005;77:18-24.

9. Höckel M, Vaupel P. Tumor hypoxia: definitions and current clinical, biologic, and molecular aspects. J Natl Cancer Inst 2001;93:266-76.

10. Lopci E, Grassi I, Chiti A, et al. PET radiopharmaceuticals for imaging of tumor hypoxia: a review of the evidence. Am J Nucl Med Mol Imaging 2014;4:365-84.

11. Rasey JS, Koh WJ, Grierson JR, et al. Radiolabelled fluoromisonidazole as an imaging agent for tumor hypoxia. Int J Radiat Oncol Biol Phys 1989;17:985-91.

12. Rasey JS, Koh WJ, Evans ML, et al. Quantifying regional hypoxia in human tumors with positron emission tomography of [18F] fluoromisonidazole: a pretherapy study of 37 patients. Int J Radiat Oncol Biol Phys 
1996;36:417-28.

13. Eschmann SM, Paulsen F, Reimold M, et al. Prognostic impact of hypoxia imaging with $18 \mathrm{~F}$-misonidazole PET in non-small cell lung cancer and head and neck cancer before radiotherapy. J Nucl Med 2005;46:253-60.

14. Rischin D, Hicks RJ, Fisher R, et al. Prognostic significance of [18F]-misonidazole positron emission tomography-detected tumor hypoxia in patients with advanced head and neck cancer randomly assigned to chemoradiation with or without tirapazamine: a substudy of Trans-Tasman Radiation Oncology Group Study 98.02. J Clin Oncol 2006;24:2098-104.

15. Lee N, Nehmeh S, Schoder H, et al. Prospective trial incorporating pre-/mid-treatment $[18 \mathrm{~F}]$-misonidazole positron emission tomography for head-and-neck cancer patients undergoing concurrent chemoradiotherapy. Int J Radiat Oncol Biol Phys 2009;75:101-8.

16. van Hagen $\mathrm{P}$, Hulshof MC, van Lanschot JJ, et al. Preoperative chemoradiotherapy for esophageal or junctional cancer. N Engl J Med 2012;366:2074-84.

17. Zhao Q, Rashid A, Gong Y, et al. Pathologic complete response to neoadjuvant therapy in patients with pancreatic ductal adenocarcinoma is associated with a better prognosis. Ann Diagn Pathol 2012;16:29-37.

18. Evans DB, Varadhachary GR, Crane CH, et al. Preoperative gemcitabine-based chemoradiation for patients with resectable adenocarcinoma of the pancreatic head. J Clin Oncol 2008;26:3496-502.

19. Iacobuzio-Donahue CA, Fu B, Yachida S, et al. DPC4 gene status of the primary carcinoma correlates with patterns of failure in patients with pancreatic cancer. J Clin Oncol 2009;27:1806-13.

20. Hammond EM, Asselin MC, Forster D, et al. The meaning, measurement and modification of hypoxia in the laboratory and the clinic. Clin Oncol (R Coll Radiol) 2014;26:277-88.

21. Gray LH, Conger AD, Ebert M, et al. The concentration of oxygen dissolved in tissues at the time of irradiation as a factor in radiotherapy. Br J Radiol 1953;26:638-48.

22. Horsman MR, Mortensen LS, Petersen JB, et al. Imaging hypoxia to improve radiotherapy outcome. Nat Rev Clin Oncol 2012;9:674-87.

23. Brink I, Imdahl A, Juettner E, et al. Metabolic differences between esophageal adeno- and squamous cell carcinomas measured with 18F-MISO and 18F-FDG PET. J Nucl Med 2006;47:229P.

24. Brink I, Baier P, Jüttner E, et al. Assessment of hypoxia in esophageal carcinomas using 18F-MISO PET. J Nucl Med
2008;49:113P.

25. Yue J, Yang Y, Cabrera AR, et al. Measuring tumor hypoxia with (1)(8)F-FETNIM PET in esophageal squamous cell carcinoma: a pilot clinical study. Dis Esophagus 2012;25:54-61.

26. Klaassen R, Bennink RJ, van Tienhoven G, et al. Feasibility and repeatability of PET with the hypoxia tracer $[(18) \mathrm{F}] \mathrm{HX} 4$ in oesophageal and pancreatic cancer. Radiother Oncol 2015;116:94-9.

27. Segard T, Robins PD, Yusoff IF, et al. Detection of hypoxia with $18 \mathrm{~F}$-fluoromisonidazole (18F-FMISO) PET/CT in suspected or proven pancreatic cancer. Clin Nucl Med 2013;38:1-6.

28. Metran-Nascente C, Yeung I, Vines DC, et al. Measurement of Tumor Hypoxia in Patients with Advanced Pancreatic Cancer Based on 18F-Fluoroazomyin Arabinoside Uptake. J Nucl Med 2016;57:361-6.

29. Yopp AC, Schwartz LH, Kemeny N, et al. Antiangiogenic therapy for primary liver cancer: correlation of changes in dynamic contrast-enhanced magnetic resonance imaging with tissue hypoxia markers and clinical response. Ann Surg Oncol 2011;18:2192-9.

30. Peerlings J, Van De Voorde L, Mitea C, et al. Hypoxia and hypoxia response-associated molecular markers in esophageal cancer: A systematic review. Methods 2017;130:51-62.

31. Mahadevan D, Von Hoff DD. Tumor-stroma interactions in pancreatic ductal adenocarcinoma. Mol Cancer Ther 2007;6:1186-97.

32. Erkan M, Reiser-Erkan C, Michalski CW, et al. Cancerstellate cell interactions perpetuate the hypoxia-fibrosis cycle in pancreatic ductal adenocarcinoma. Neoplasia 2009;11:497-508.

33. Koong AC, Mehta VK, Le QT, et al. Pancreatic tumors show high levels of hypoxia. Int J Radiat Oncol Biol Phys 2000;48:919-22.

34. Kasuya K, Tsuchida A, Nagakawa Y, et al. Hypoxiainducible factor- $1 \alpha$ expression and gemcitabine chemotherapy for pancreatic cancer. Oncol Rep 2011;26:1399-406.

35. Wong KM, Horton KJ, Coveler AL, et al. Targeting the Tumor Stroma: the Biology and Clinical Development of Pegylated Recombinant Human Hyaluronidase (PEGPH20). Curr Oncol Rep 2017;19:47.

36. Klaassen R, Gurney-Champion OJ, Wilmink JW, et al. Repeatability and correlations of dynamic contrast enhanced and T2* MRI in patients with advanced pancreatic ductal adenocarcinoma. Magn Reson Imaging 
2018;50:1-9.

37. Wegner CS, Hauge A, Gaustad JV, et al. Dynamic contrast-enhanced MRI of the microenvironment of pancreatic adenocarcinoma xenografts. Acta Oncol 2017;56:1754-62.

38. Zhang X, Wojtkowiak JW, Martinez GV, et al. MR Imaging Biomarkers to Monitor Early Response to Hypoxia-Activated Prodrug TH-302 in Pancreatic Cancer Xenografts. PLoS One 2016;11:e0155289.

39. Riedl CC, Brader P, Zanzonico P, et al. Tumor hypoxia imaging in orthotopic liver tumors and peritoneal metastasis: a comparative study featuring dynamic 18F-MISO and 124I-IAZG PET in the same study cohort. Eur J Nucl Med Mol Imaging 2008;35:39-46.

40. Øvrebø KM, Hompland T, Mathiesen B, et al. Assessment of hypoxia and radiation response in intramuscular experimental tumors by dynamic contrast-enhanced magnetic resonance imaging. Radiother Oncol 2012;102:429-35.

41. Egeland TA, Gulliksrud K, Gaustad JV, et al. Dynamic contrast-enhanced-MRI of tumor hypoxia. Magn Reson Med 2012;67:519-30.

42. Lee N, Schoder H, Beattie B, et al. Strategy of Using Intratreatment Hypoxia Imaging to Selectively and Safely Guide Radiation Dose De-escalation Concurrent With Chemotherapy for Locoregionally Advanced Human Papillomavirus-Related Oropharyngeal Carcinoma. Int J Radiat Oncol Biol Phys 2016;96:9-17.

43. Kong FM, Ten Haken RK, Schipper M, et al. Effect of Midtreatment PET/CT-Adapted Radiation Therapy With Concurrent Chemotherapy in Patients With Locally Advanced Non-Small-Cell Lung Cancer: A Phase 2 Clinical Trial. JAMA Oncol 2017;3:1358-65.

44. Bradley JD, Paulus R, Komaki R, et al. Standard-dose versus high-dose conformal radiotherapy with concurrent and consolidation carboplatin plus paclitaxel with or without cetuximab for patients with stage IIIA or IIIB non-smallcell lung cancer (RTOG 0617): a randomised, two-by-two factorial phase 3 study. Lancet Oncol 2015;16:187-99.

45. Dehdashti F, Grigsby PW, Lewis JS, et al. Assessing tumor hypoxia in cervical cancer by PET with 60Cu-labeled diacetyl-bis(N4-methylthiosemicarbazone). J Nucl Med 2008;49:201-5.

46. Christian N, Deheneffe S, Bol A, et al. Is (18)F-FDG a surrogate tracer to measure tumor hypoxia? Comparison with the hypoxic tracer (14)C-EF3 in animal tumor models. Radiother Oncol 2010;97:183-8.

47. Bentzen SM, Gregoire V. Molecular imaging-based dose painting: a novel paradigm for radiation therapy prescription. Semin Radiat Oncol 2011;21:101-10.

48. Popple RA, Ove R, Shen S. Tumor control probability for selective boosting of hypoxic subvolumes, including the effect of reoxygenation. Int J Radiat Oncol Biol Phys 2002;54:921-7.

49. Hendrickson K, Phillips M, Smith W, et al. Hypoxia imaging with [F-18] FMISO-PET in head and neck cancer: potential for guiding intensity modulated radiation therapy in overcoming hypoxia-induced treatment resistance. Radiother Oncol 2011;101:369-75.

50. Minsky BD, Pajak TF, Ginsberg RJ, et al. INT 0123 (Radiation Therapy Oncology Group 94-05) phase III trial of combined-modality therapy for esophageal cancer: high-dose versus standard-dose radiation therapy. J Clin Oncol 2002;20:1167-74.

51. Krishnan S, Chadha AS, Suh Y, et al. Focal Radiation Therapy Dose Escalation Improves Overall Survival in Locally Advanced Pancreatic Cancer Patients Receiving Induction Chemotherapy and Consolidative Chemoradiation. Int J Radiat Oncol Biol Phys 2016;94:755-65.

52. Tao R, Krishnan S, Bhosale PR, et al. Ablative Radiotherapy Doses Lead to a Substantial Prolongation of Survival in Patients With Inoperable Intrahepatic Cholangiocarcinoma: A Retrospective Dose Response Analysis. J Clin Oncol 2016;34:219-26.

53. Moertel CG, Frytak S, Hahn RG, et al. Therapy of locally unresectable pancreatic carcinoma: a randomized comparison of high dose (6000 rads) radiation alone, moderate dose radiation (4000 rads +5 -fluorouracil), and high dose radiation +5 -fluorouracil: The Gastrointestinal Tumor Study Group. Cancer 1981;48:1705-10.

54. Crane CH, Macdonald KO, Vauthey JN, et al. Limitations of conventional doses of chemoradiation for unresectable biliary cancer. Int J Radiat Oncol Biol Phys 2002;53:969-74.

55. Hong TS, Wo JY, Yeap BY, et al. Multi-Institutional Phase II Study of High-Dose Hypofractionated Proton Beam Therapy in Patients With Localized, Unresectable Hepatocellular Carcinoma and Intrahepatic Cholangiocarcinoma. J Clin Oncol 2016;34:460-8.

Cite this article as: Tao R, Ager B, Lloyd S, Torgeson A, Denney M, Gaffney D, Kharofa J, Lin SH, Koong AC, Anzai Y, Hoffman JM. Hypoxia imaging in upper gastrointestinal tumors and application to radiation therapy. J Gastrointest Oncol 2018;9(6):1044-1053. doi: 10.21037/jgo.2018.09.15 